\title{
Dynamical transition for a 3-component Lotka-Volterra Model with Diffusion
}

\author{
Ruili $\mathrm{Wu}^{1}$, Limei $\mathrm{Li}^{2}$, and Junyan $\mathrm{LI}^{3}$ \\ ${ }^{1}$ Jincheng College of Sichuan University \\ ${ }^{2}$ Sichuan Normal University \\ ${ }^{3}$ Jincheng college of Sichuan University
}

August 13, 2020

\begin{abstract}
The main objective of this article is to investigate the dynamical transition for a 3-component Lotka-Volterra model with diffusion. Based on the spectral analysis, the principle of exchange of stability conditions for eigenvalues are obtained. In addition, when $\$ \backslash$ delta_ $0<\backslash$ delta_ $1 \$$, the first eigenvalues are complex, and we show that the system undergoes a continuous or jump transition. In the small oscillation frequency limit, the transition is always continuous and the time periodic rolls are stable after the transition. In the case where $\$ \backslash$ delta_ $0>\backslash$ delta_ $1 \$$, the first eigenvalue is real. Generically, the first eigenvalue is simple and all three types of transition are possible. In particular, the transition is mixed if $\$ \backslash$ int_$_{-}\{\backslash$ Omega $\} \mathrm{e}_{-}\left\{\mathrm{k}_{-} 0\right\}^{\wedge} 3 \mathrm{dx} \backslash$ neq $0 \$$, and is continuous or jump in the case where $\$ \backslash \operatorname{int}_{-}\{\backslash$ Omega $\} \mathrm{e}_{-}\left\{\mathrm{k}_{-} 0\right\}^{\wedge} 3 \mathrm{dx}=0 \$$. In this case we also show that the system bifurcates to two saddle points on $\$ \backslash$ delta $<\backslash$ delta_ $1 \$$ as $\$ \backslash$ tilde $\{\backslash$ theta $\}>0 \$$, and bifurcates to two stable singular points on $\$ \backslash$ delta $>\backslash$ delta_ $1 \$$ as $\$ \backslash$ tilde $\{\backslash$ theta $\}<0 \$$ where $\$ \backslash$ tilde $\{\backslash$ theta $\} \$$ depends on the system parameters.
\end{abstract}

\section{Hosted file}

Wu_LI_LI.pdf available at https://authorea.com/users/310868/articles/475466-dynamicaltransition-for-a-3-component-lotka-volterra-model-with-diffusion

\section{Hosted file}

Wu_LI_LI.tex available at https://authorea.com/users/310868/articles/475466-dynamicaltransition-for-a-3-component-lotka-volterra-model-with-diffusion

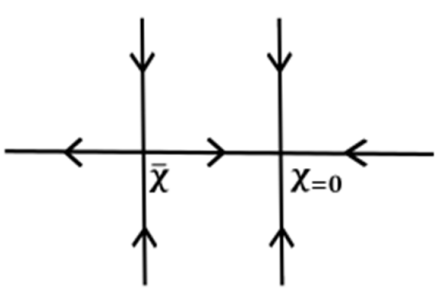

(1)

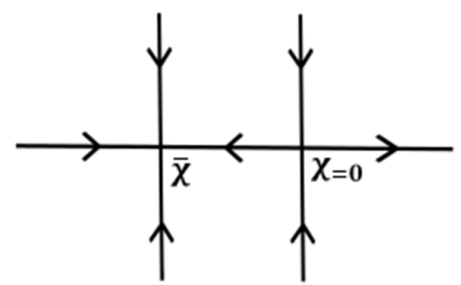

(2) 


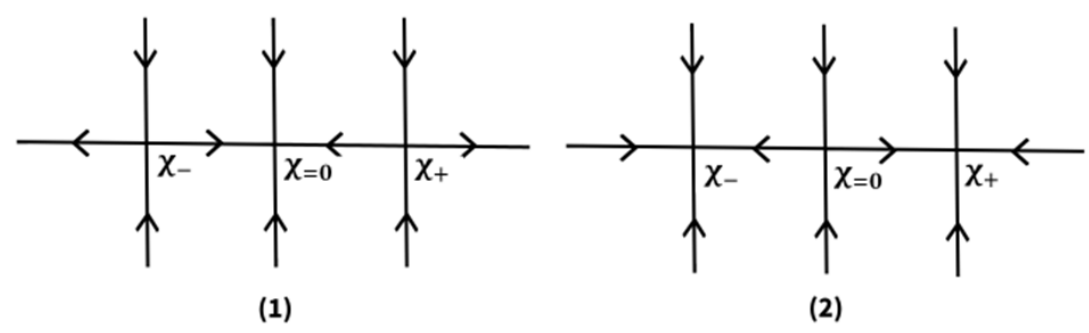

2 